\title{
Cluster Core-Level Binding-Energy Shifts: The Role of Lattice Strain
}

\author{
B. Richter, ${ }^{1}$ H. Kuhlenbeck, ${ }^{1}$ H.-J. Freund, ${ }^{1}$ and P. S. Bagus ${ }^{2}$ \\ ${ }^{1}$ Fritz-Haber-Institut der Max-Planck-Gesellschaft, Faradayweg 4-6, D-14195, Berlin, Germany \\ ${ }^{2}$ Department of Chemistry, University of North Texas, Denton, Texas 76203-5070, USA
}

(Received 30 April 2003; published 8 July 2004)

\begin{abstract}
Our combined experimental and theoretical analysis of the shifts, with particle size, of core-level binding energies (BE's) of metal nanoparticles on insulating supports, shows that these shifts have an important initial state contribution arising, in large part, because of lattice strain. This contribution of $\mathrm{BE}$ shifts has not been recognized previously. Lattice strain changes the chemical bonding between the metal atoms and this change induces BE shifts.
\end{abstract}

DOI: 10.1103/PhysRevLett.93.026805

PACS numbers: 73.22.-f, 79.60.-i

Photoelectron spectroscopy (PES) is often used to deduce information on the electronic structure of molecules, solids, and at surfaces [1]. There is increasing interest in nanostructured materials, especially in clusters grown on inert surfaces since they may have high catalytic activity [2]. Thus, the question of how the PES of deposited nanoparticles reflects their electronic and geometric structures is quite important. It is known that the corelevel BE's of metal clusters on insulating supports [3-12] shift to lower $\mathrm{BE}$ with increasing size by $\Delta \mathrm{BE} \sim 1 \mathrm{eV}$ from small clusters to bulk metal. However, there is substantial disagreement over the assignment of these shifts to initial or final state effects; see Ref. [13] for a rigorous definition of these two contributions. Mason [7] argued that changes in the electron configuration of the atoms in smaller clusters, not relaxation energies, were primarily responsible for the shift. On the other hand, Wertheim and collaborators $[4,5]$ assigned the shift as due to final state screening effects and related the magnitude of $\triangle \mathrm{BE}$ to an effective cluster radius. For $\mathrm{Cu}$ clusters grown on thin $\mathrm{Al}_{2} \mathrm{O}_{3}$ films, Wu et al. [11] concluded from an Auger parameter analysis that initial state contributions are small and may be significant only for very small clusters. This separation is quite important since the initial state $\triangle \mathrm{BE}$ reflect changes in the electronic structure before ionization of a core level and, hence, are of direct interest for materials properties. In the present work, we show that the initial and final state contributions to the $\triangle \mathrm{BE}$ are of comparable magnitude. Further, we relate the initial state $\Delta \mathrm{BE}$ to a lattice strain that exists because the average bond distances in small clusters are shorter than in the bulk $[14,15]$. The chemical bonding is different at the shorter bond distances and this, in turn, leads to changes in the core-level BE's. The chemical changes important for the $\triangle \mathrm{BE}$ involve an increased $d$ to $s p$ promotion, or hybridization, for shorter bond distances.

Our work is based on a combination of experimental and theoretical methods that allow us to decompose the $\triangle \mathrm{BE}$ into initial and final state contributions. Our theoretical approach involves the calculation of electronic wave functions (WF's) for clusters and the determination of the BE's where relaxation in response to ionization is excluded, a pure initial state $\mathrm{BE}$, and where this relaxation is allowed [13]. Our experimental approach involves use of an Auger parameter obtained from measured Auger kinetic energies and core-level BE's. The Auger parameter concept, developed by Wagner [16], has been discussed by several authors $[3,17-21]$. We use the extension proposed by Hohlneicher et al. [21], where the two Auger final state core holes are in the same shell; this extension puts the Auger parameter analysis on a sound foundation. Previous applications of Auger parameter analysis to the $\triangle \mathrm{BE}$ of supported clusters [11,22] have not used these refinements [21]. Both the Auger parameter analysis and the WF based separation of initial and final state contributions to the $\triangle \mathrm{BE}$ with cluster size show that these two terms have large magnitudes.

The experiments were performed at the BESSY II synchrotron facility in Berlin. We studied Co, rather than $\mathrm{Cu}$ [11], clusters on $\mathrm{Al}_{2} \mathrm{O}_{3}$ films for two reasons. First, it is easier to control particle size for Co since it is less mobile than $\mathrm{Cu}$ [23]. Second, it is possible to study the Co $L_{3} M_{2,3} M_{2,3}$ Auger transition where all holes are in the core, while for $\mathrm{Cu}$ the best results reported [11] used the $L_{3} M_{4,5} M_{4,5}$ Auger lines where the $\mathrm{Cu} 3 d, M_{4,5}$, electrons participate in the chemical bonding. However, we expect that the results for $\mathrm{Co} / \mathrm{Al}_{2} \mathrm{O}_{3}$ are representative for metal nanoparticles on relatively inert substrates [7]. The ultrahigh vacuum system consists of two chambers, one used for preparation purposes, the other analysis chamber carries a SCIENTA electron spectrometer as well as detectors to perform $\mathrm{x}$-ray fluorescence measurements [24]. The analysis chamber is connected to an undulator beam line (U 49) equipped with a plane grating monochromator, yielding a resolution of approximately $10^{4}$, and a photon flux near $10^{11}$ photons/s. The sample, a $\mathrm{NiAl}(110)$ single crystal, is mounted on a manipulator which allows for translation between the chambers. The sample can be heated and cooled. The preparation of the alumina film uses established recipes [25]. Co is evaporated from an electron beam evaporator (focus 
EFM4) at a rate of $0.5 \AA$ per minute. In separate STM experiments, the film morphology has been determined. By combining the island density as determined by STM and the deposited mass from the quartz balance, the average number of Co atoms per cluster is obtained [26].

Figure 1 shows photoelectron and Auger spectra of deposited Co aggregates for several coverages. With the data for the shifts of the Co $3 p$ and $2 p_{3 / 2}$ BE's and the $L_{3} M_{2,3} M_{2,3}$ Auger energies, we perform an Auger parameter analysis. The initial and final state contributions to the $\triangle \mathrm{BE}$ obtained from this analysis are plotted in Fig. 2 as a function of particle size. The initial state shift is represented by $\Delta \varepsilon$, the change in an effective orbital energy. From Koopmans' Theorem, the initial state BE = $-\varepsilon[3,13]$; thus $\Delta \varepsilon<0$ indicates a shift to higher BE. The final state, relaxation energy, contribution to the $\Delta \mathrm{BE}$ is denoted $\Delta R$, and $\Delta R<0$ indicates a shift to higher BE. In Fig. 2, $\Delta \varepsilon$ and $\Delta R$, measured with respect to bulk BE's, are both negative. The mean particle radius is obtained assuming the particles are half spheres, consistent with our STM profiles [24]. For the shift between the smallest clusters, $\sim 4.7 \AA$ radius, and thick Co films, the Auger parameter analysis gives an initial state contribution that is $31 \%$ of the total $\triangle \mathrm{BE}=0.98 \mathrm{eV}$. Clearly, initial state effects make a major contribution.

The goal of our theoretical studies of the $\triangle \mathrm{BE}$ is to obtain an understanding of the physical mechanisms responsible for the trend of the decrease of $\mathrm{BE}$ with increasing particle size, observed for a variety of metals [7]. Since we do not intend to simulate the explicit dependence of the $\triangle \mathrm{BE}$ on particle size, we are able to make approximations to simplify these studies: (i) We study isolated clusters and neglect the weak interaction of the particles with the oxide substrate [7]. (ii) We study $\mathrm{Cu}$ clusters in order to avoid computational difficulties related to open $d$ shells. For a variety of noble and transition

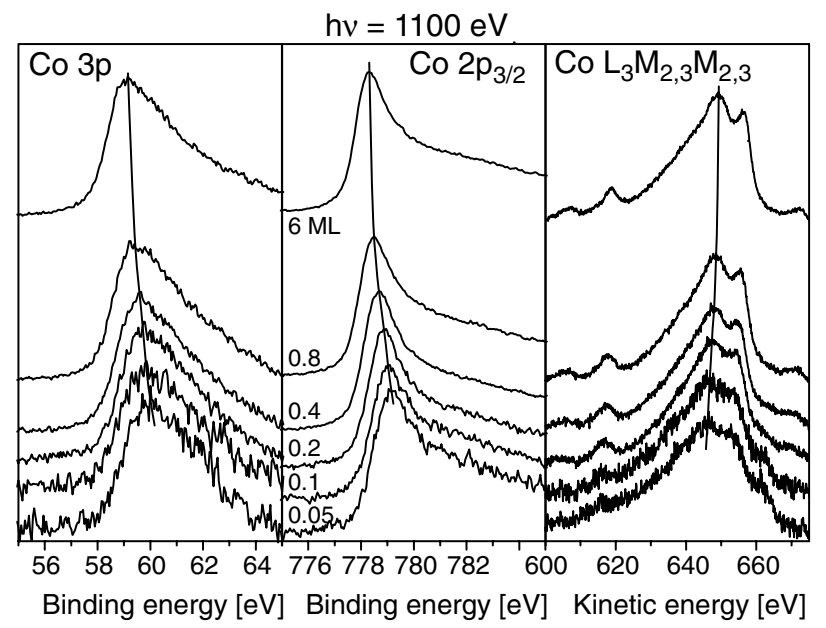

FIG. 1. The Co $3 p$ and the Co $2 p_{3 / 2}$ core-level BE's and the Co $L_{3} M_{2,3} M_{2,3}$ Auger transitions for Co clusters deposited onto $\mathrm{Al}_{2} \mathrm{O}_{3} / \mathrm{NiAl}(110)$ as a function of coverage; lines are drawn to show shifts with coverage metal particles, the BE shift with particle size is $\sim 1 \mathrm{eV}$ [3-12]; thus, it seems appropriate to study the $\triangle \mathrm{BE}$ for $\mathrm{Cu}$. However, we also study $\triangle \mathrm{BE}$ for small Ni clusters to confirm the generality, for open $d$-shell metals, of our results for $\mathrm{Cu}$. (iii) The geometric structure of all $\mathrm{Cu}$ and $\mathrm{Ni}$ clusters studied was taken as that of the fcc crystal structure and the lattice strain is modeled by uniform changes in the lattice constant, $a_{0}$. This model does not explicitly treat the specific shapes of the supported particles. (iv) Given our idealized cluster geometries, we consider only BE's for cluster atoms that have the bulk coordination. Although core-level BE's do depend on the coordination of the ionized atom [13], this dependence, especially for transition and noble metal atoms, is relatively weak; for example, the BE shifts between surface and bulk atoms of metal crystals are not large, $\sim 0.25 \mathrm{eV}$ [3]. Based on our results for the BE shifts with lattice strain for (100) surface atoms compared to the $\triangle \mathrm{BE}$ for bulk atoms, we expect the contributions of differently coordinated atoms in supported particles to lead, dominantly, to broadening of the PES peaks.

Initial and final state contributions to the $\triangle \mathrm{BE}$ were determined for a series of clusters chosen to model both particle size and lattice strain effects. The initial state BE's are obtained with Hartree-Fock self-consistent-field (SCF) molecular orbitals for the ground state of the cluster before ionization. These orbitals are used to form a frozen orbital (FO) WF for the ionic states where relaxation to screen the core hole is not allowed. The difference between the energies of the ground state and the FO ionic state WF's is BE(initial). The difference between the energies of the ground state and the SCF WF for the ionized state $[\mathrm{BE}(\triangle \mathrm{SCF})]$ is the total $\mathrm{BE}$. The SCF orbitals for the ionic state WF's, optimized for the presence of the core hole, fully include electronic relaxation in response to the hole [27]. The change between the initial and total BE's is the relaxation energy, $E_{R}$, associated with the final state effects;

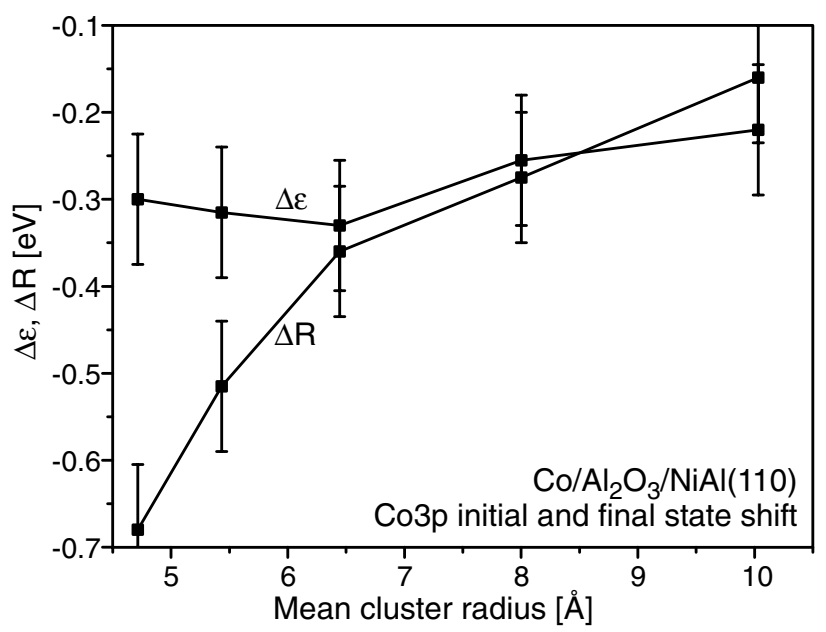

FIG. 2. Co $3 p$ initial $(\Delta e)$ and final state $(\Delta R)$ shifts as a function of the mean cluster radius. 
$E_{R}=\mathrm{BE}($ initial $)-\mathrm{BE}(\Delta \mathrm{SCF}) . \quad$ The shifts are: $\triangle \mathrm{BE}(\triangle \mathrm{SCF})$ for the changes due to both initial and final state effects; $\triangle \mathrm{BE}$ (initial) for the initial state changes; and $\Delta E_{R}$. Their relationship to the quantities obtained in the Auger parameter analysis is $\Delta \mathrm{BE}($ initial $)=-\Delta \varepsilon$ and $\Delta E_{R}=\Delta R$. However, the quantities obtained from the SCF WF's rigorously separate initial and final state contributions [13], while the Auger parameter separation involves assumptions about the intra- and extra-atomic relaxation [16-21]. Furthermore, the SCF energies of double-hole Auger states can be used to calculate the Auger parameter $\beta$ [21] and the Auger relationship that $\Delta E_{R}=\Delta \beta / 2$ can be compared to the $\Delta E_{R}$ calculated directly from $\mathrm{BE}$ (initial) and $\mathrm{BE}(\Delta \mathrm{SCF})$.

The clusters used to study the progression of BE's toward the bulk value are shown in Fig. 3. The largest, $\mathrm{Cu}_{115}$, cluster has seven layers parallel to the (100) surface; the numbers of atoms in each layer are $\mathrm{Cu}_{115}(4,25,16,25,16,25,4)$. We use the BE's for $\mathrm{Cu}_{115}$ to represent the limit of a large cluster although the BE's for clusters of this size are not fully converged to the bulk [24]. The next smaller cluster, $\mathrm{Cu}_{55}(9,12,12,12,9)$, has four layers and the smallest cluster, $\mathrm{Cu}_{18}(5,4,5,4)$, has four layers. For $\mathrm{Cu}_{115}$ and $\mathrm{Cu}_{55}$, we consider BE's for ionization of the central atom; for $\mathrm{Cu}_{18}$, the $\mathrm{BE}$ 's are for ionization of the central atom of the third layer. In order to study only the effect of cluster size on the BE's, we have used the bulk $a_{0}=3.59 \AA$. However, there is evidence from transmission electron microscopy of $\mathrm{Pd}$ and $\mathrm{Pt}$ on $\mathrm{Al}_{2} \mathrm{O}_{3}$ that the effective $a_{0}$ for small clusters is reduced by $\sim 5 \%-7 \%[14,15]$; these contractions are much larger than those found for matrix isolated $\mathrm{Cu}$ clusters [28]. The much larger lattice strain for supported clusters may be due to their weak interaction with the support. The effect of this lattice strain on the core-level BE's is studied by determining the BE's for $\mathrm{Cu}_{18}$ with three reductions of $a_{0}$ to $3.52,3.44$, and $3.37 \AA$. For atoms where core level BE's may be studied, all the $29 \mathrm{Cu}$ electrons are explicitly included in the cluster WF's; for the other atoms, the core electrons are represented by a pseudopotential [13]. For the open shell $\mathrm{Cu}_{55}$ and $\mathrm{Cu}_{115}$ clusters, we consider BE's to the weighted averages of the

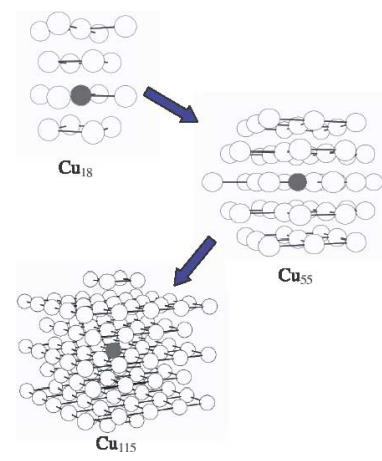

FIG. 3 (color online). Size progression of $\mathrm{Cu}_{18}$ to $\mathrm{Cu}_{55}$ to $\mathrm{Cu}_{115}$ clusters. The ionized atom is shaded. ionic states. To avoid the distraction of spin-orbit splittings in the $2 p$ shell, we report here shifts of the $2 s$ BE's; the $\triangle \mathrm{BE}$ 's calculated for $1 s$ and $3 s$ holes are very similar to the $\Delta \mathrm{BE}(2 s)$. For the theoretical determination of $\beta(2 s)$ [21], we use SCF WF's for the $2 s$ and $3 s$ hole states and for the $3 s$ double hole Auger state. We also report $\Delta \mathrm{BE}(2 s)$ for $\mathrm{Ni}_{18}$ clusters constructed in an analogous way to the $\mathrm{Cu}_{18}$ clusters; the lattice strain for $\mathrm{Ni}$ is examined by using contracted $a_{0}$ of $3.45,3.37$, and $3.30 \AA$ as well as bulk $a_{0}=3.52 \AA$.

In Table I, we give $\Delta \mathrm{BE}(\Delta \mathrm{SCF}), \Delta \mathrm{BE}\left(\right.$ initial), and $\Delta E_{R}$ at the bulk $a_{0}$ and, for $\mathrm{Cu}_{18}$, also at a representative contracted $a_{0}=3.44 \AA$. We also give shifts of the Auger parameter, $\Delta \beta / 2$. The sequence from $\mathrm{Cu}_{18}\left(a_{0}=\right.$ $3.44 \AA)$ to $\mathrm{Cu}_{115}\left(a_{0}=3.59 \AA\right)$ models the progression of $\triangle \mathrm{BE}$ from small clusters to bulk; the shift to lower BE of $\sim 1 \mathrm{eV}$ is consistent with the measured values for BE shifts in supported clusters and indicates that our cluster models correctly describe the physics of this shift. Note that the total shift has approximately equal contributions from initial and final state shifts.

Although the increase of the bond distance from small to large clusters is not a step function, we use the step from $\mathrm{Cu}_{18}\left(a_{0}=3.44 \AA\right)$ to $\mathrm{Cu}_{18}\left(a_{0}=3.59 \AA\right)$ to separate the increase of the bond distance from the change of the number of atoms. The increase of $a_{0}$ leads to a shift of $0.5 \mathrm{eV}$ to lower BE, $\sim 50 \%$ of the total $\Delta \mathrm{BE}$ between small clusters and bulk; this shift is almost entirely an initial state effect (see Table I). There is a further large BE decrease of $0.67 \mathrm{eV}$ when the cluster size is increased from $\mathrm{Cu}_{18}$ to $\mathrm{Cu}_{115}$ with bulk $a_{0}$ that is $\sim 2 / 3$ due to an increase in final state relaxation and $\sim 1 / 3$ to an initial state BE shift. In other words, the final state relaxation dominates the shift to lower BE with increasing cluster size for a constant $a_{0}$; this is consistent with a monotonic dependence of $E_{R}$ on system size $[3,6,13]$. The $\triangle \mathrm{BE}$ (initial) do not vary monotonically with cluster size suggesting that there is a dependence of the initial state $\mathrm{BE}$ on the cluster morphology. However, the dependence of $\mathrm{BE}$ (initial) on the details of the cluster morphology is not particularly large and is not investigated further.

For $\mathrm{Ni}_{18}$ and $\mathrm{Cu}_{18}$, the dependence of the $2 s$ BE's on $a_{0}$ is shown in Table II. For both metals, the $\Delta \mathrm{BE}(\triangle \mathrm{SCF})$, and $\triangle B E$ (initial) are almost the same and vary nearly linearly with $\Delta a_{0}$; these changes of $\Delta \mathrm{BE}$ with $a_{0}$ show

TABLE I. The $2 s$ BE shifts for $\mathrm{Cu}_{n}$ at bulk $a_{0}$ with respect to $\Delta \mathrm{BE}\left(\mathrm{Cu}_{115}\right)=0$; for $\mathrm{Cu}_{18}$, a reduced $a_{0}$ is also used. $\Delta E_{R}$ and $\Delta \beta / 2$ are shown. All energies are in $\mathrm{eV}$.

\begin{tabular}{lcccc}
\hline \hline & $\mathrm{Cu}_{18}$ & $\mathrm{Cu}_{18}$ & $\mathrm{Cu}_{55}$ & $\mathrm{Cu}_{115}$ \\
\multicolumn{1}{c}{$a_{0}-\AA$} & 3.44 & 3.59 & 3.59 & 3.59 \\
\hline$\Delta \mathrm{BE}(\Delta \mathrm{SCF})$ & +1.21 & +0.67 & +0.34 & 0 \\
$\Delta \mathrm{BE}($ initial $)$ & +0.73 & +0.22 & +0.27 & 0 \\
$\Delta E_{R}$ & -0.47 & -0.45 & -0.07 & 0 \\
$\Delta \beta / 2$ & -0.67 & -0.66 & -0.21 & 0 \\
\hline \hline
\end{tabular}


TABLE II. $\Delta \mathrm{BE}(\Delta \mathrm{SCF})$ and $\triangle \mathrm{BE}$ (initial), in $\mathrm{eV}$, for the $\mathrm{Cu}$ and $\mathrm{Ni} 2 s$ BE's in 18 atom clusters. The changes in the lattice constants are given as $\Delta a_{0}$ in percent change from bulk $a_{0}$. $\Delta \mathrm{BE}=0$ for $\Delta a_{0}=0$. For $\mathrm{Cu}$, frozen core and frozen Ar core values of $\Delta \mathrm{BE}$ (initial) are given (see text).

\begin{tabular}{lcccc}
\hline \hline \multicolumn{1}{c}{$\Delta a_{0}$} & $-6 \%$ & $-4 \%$ & $-2 \%$ & 0 (bulk) \\
\hline $\mathrm{Cu}$ & & & & \\
$\Delta \mathrm{BE}(\Delta \mathrm{SCF})$ & +0.84 & +0.54 & +0.26 & 0 \\
$\Delta \mathrm{BE}($ initial $)$ & +0.79 & +0.51 & +0.25 & 0 \\
Frozen core & +0.12 & +0.05 & +0.01 & 0 \\
Frozen Ar core & +0.78 & +0.51 & +0.24 & 0 \\
$\mathrm{Ni}$ & & & & \\
$\Delta \mathrm{BE}(\Delta \mathrm{SCF})$ & +0.72 & +0.45 & +0.21 & 0 \\
$\Delta \mathrm{BE}($ initial $)$ & +0.73 & +0.47 & +0.22 & 0 \\
\hline \hline
\end{tabular}

the generality of the dependence of the BE's on lattice strain. The near equality of the initial and $\triangle \mathrm{SCF}$ values show that the increase of the BE's due to lattice contraction is a dominantly initial state effect. The origin of the large $\triangle \mathrm{BE}$ (initial) is found by separating, using constrained variations [13], the contributions due to the $4 s p$ conduction band electrons from those due to the $3 d$ electrons. This decomposition is given only for $\mathrm{Cu}$ but it is similar for Ni. For the frozen core WF, the orbitals of the electrons in the $1 s$ to $3 d$ shells are fixed to be the same as in the isolated atom. With this constraint, the $\mathrm{BE}$ (initial) depend on $a_{0}$ only because of changes in the conduction band, $4 s p$ electrons. For the frozen Ar core WF, only the $18 \mathrm{Ar}$ core electrons are constrained to be atomic. Now, $3 d$ hybridization and bonding is allowed. It is clear that the $3 d$ chemistry is the main reason for the large $\Delta \mathrm{BE}$ as $a_{0}$ is reduced. The hybridization and promotion of a fraction of an electron from the contracted $3 d$ shell into a more diffuse orbital leads to a large increase in the core-level BE's [13]. The $d$ hybridization increases strongly for shorter $a_{0}$ since shorter bond distances favor an increased bonding participation of the compact $d$ orbitals.

The changes of the Auger parameter, $\Delta \beta / 2$, roughly follow those of the $\Delta E_{R}$; see Table I. The values of $\Delta \beta / 2$ are essentially equal for $\mathrm{Cu}_{18}$ with $a_{0}=3.44$ and $3.59 \AA$; the same near equality is also found for $\Delta E_{R}$. From $\mathrm{Cu}_{18}$ with bulk $a_{0}$ to $\mathrm{Cu}_{115}$, the increases of $\Delta \beta / 2$ parallel the increases of $\Delta E_{R}$; however, the increases of $\Delta \beta / 2$ are larger than those of $\Delta E_{R}$. While the trend of $\Delta E_{R}$ is correctly described by the trend of $\Delta \beta / 2$, the Auger parameter analysis appears to indicate a larger contribution from final state effects than given by the $a b$ initio decomposition of the BE's. This problem may be related to assumptions about the intra- and extra-atomic contributions to $E_{R}$ made in the derivation of the Auger parameter relationship.

A large initial state contribution to the cluster size dependent BE shifts has been shown from both Auger parameter analysis of the experimental data and from calculation of the $\Delta \mathrm{BE}$ with ab initio cluster WF's leaving no doubt that initial state contributions are significant. Further, we have shown that the origin of the initial state effect is a lattice contraction that is part of the cluster growth morphology $[14,15]$. In particular, the BE shift is related to the $d$ hybridization being larger for shorter bond distances. These are important extensions of our understanding of the origin and the physical significance of the cluster BE shifts.

We acknowledge partial computer support from the National Center for Supercomputing Applications, Urbana-Champaign, Illinois.

[1] S. Hüfner, Photoelectron Spectroscopy: Principles and Applications (Springer-Verlag, Berlin, 1995).

[2] C. T. Campbell, Surf. Sci. Rep. 27, 1 (1997).

[3] W. F. Egelhoff, Jr., Surf. Sci. Rep. 6, 253 (1987).

[4] G. K. Wertheim, Z. Phys. B 66, 53 (1987); Z. Phys. D 12, 319 (1989).

[5] G. K. Wertheim, S. B. DiCenzo, and D. N. E. Buchanan, Phys. Rev. B 33, 5384 (1986).

[6] F. Parmigiani et al., J. Electron Spectrosc. Relat. Phenom. 50, 39 (1990).

[7] M. G. Mason, Phys. Rev. B 27, 748 (1983).

[8] M. Cini et al., Phys. Rev. B 41, 5685 (1990).

[9] N. Mårtensson, J. Electron Spectrosc. Relat. Phenom. 75, 209 (1995).

[10] J. A. D. Matthew, Solid State Commun. 73, 179 (1990).

[11] Y. Wu, E. Garfunkel, and T. E. Madey, J. Vac. Sci. Technol. A 14, 1546 (1996).

[12] A. Sandell et al., J. Vac. Sci. Technol. A 14, 1546 (1996).

[13] P.S. Bagus et al., J. Electron Spectrosc. Relat. Phenom. 100, 215 (1999).

[14] S. A. Nepijko et al., Langmuir 15, 5309 (1999).

[15] M. Klimenkov et al., Surf. Sci. 391, 27 (1997).

[16] See, for example, C. D. Wagner and J. A. Taylor, J. Electron Spectrosc. Relat. Phenom. 28, 211 (1982).

[17] S. P. Kowalczyk et al., Phys. Rev. B 9, 381 (1974).

[18] T. D. Thomas, J. Electron Spectrosc. Relat. Phenom. 20, 117 (1980).

[19] G. Moretti, J. Electron Spectrosc. Relat. Phenom. 95, 95 (1998).

[20] R. N. Sodhi, and R. G. Cavell, J. Electron Spectrosc. Relat. Phenom. 32, 283 (1983).

[21] G. Hohlneicher, H. Pulm, and H.-J. Freund, J. Electron Spectrosc. Relat. Phenom. 37, 209 (1985).

[22] G. K. Wertheim, Phys. Rev. B 36, 9559 (1987).

[23] M. Heemeier, Ph.D. thesis, Freie Universität Berlin, Berlin (to be published).

[24] B. Richter, Ph.D. thesis, Humboldt University, Berlin, 2002.

[25] R. M. Jaeger et al., Surf. Sci. 259, 235 (1991).

[26] M. Bäumer and H.-J. Freund, Prog. Surf. Sci. 61, 127 (1999).

[27] P. S. Bagus, Phys. Rev. 139, A619 (1965).

[28] P. A. Montano et al., Phys. Rev. Lett. 56, 2076 (1986). 